\title{
Some techniques for mass production of textile wireless systems
}

\author{
Hendrik Rogier*, Marco Rossi, Dries Van Baelen, Sam Lemey, Jo Verhaevert and Sam Agneessens \\ Ghent University-IMEC, IDLab-EM group, Dept. of Information Technology, Technologiepark Zwijnaarde 15, 9052 Gent, \\ Belgium, hendrik.rogier@ugent.be, http://emweb.intec.ugent.be/
}

\begin{abstract}
The accurate, reliable and cost-effective mass production of textile wireless systems remains an important challenge and a major impediment to achieve market penetration. Therefore, we propose some novel design, characterization and manufacturing procedures to make the development of such systems more compatible with mass production in traditional assembly line processes. First, we propose a novel non-destructive characterization procedure that determines the dielectric properties of the textile substrate directly within the assembly line, just before manufacturing the textile wireless node. Then, we describe an innovative manufacturing procedure that requires less manipulations and yields more accurate ultra-wideband textile antennas. Finally we introduce a holistic design framework for autonomous textile wireless nodes with communication, sensing and energy-harvesting functionality.
\end{abstract}

\section{Introduction}

Although textile antennas have been intensively researched for more than a decade now [1], they have not found their way to the consumer market yet. Reliability, lack of norms and standards, and incompatibilities between production processes for textiles and electronic components are a few of the reasons why the breakthrough of fullytextile wearable systems has not occurred yet. In the last years, researchers have worked hard to clear the last obstacles that impede textile electronics from coming to the market. Novel weaving techniques have resulted in improved accuracy [2] while pervasive integration of textile antenna with on-board electronic circuitry and energy harvesters [3] has led to more compact and more reliable wireless textile nodes. Yet, textile manufacturers and electronics companies remain reluctant to invest in smart textiles, due to a lack of manufacturing procedures suitable for mass production.

In this contribution, we introduce some innovative design, characterization and manufacturing strategies for mass production of smart fabric interactive textile (SFIT) systems by traditional assembly line processes. In Section 2, we outline a novel non-destructive procedure for on-the-fly dielectric property characterization of the textile substrate, directly within the assembly line. This method accurately determines the permittivity and loss tangent of each substrate sample, just before assembly into the textile wireless node. This allows adapting the design just before fabrication to compensate for variations in the material properties. Moreover, to reduce fabrication costs and dimensional variations, we describe in Section 3 a novel ultrawideband cavity-backed antenna topology that is realized by an innovative manufacturing procedure that requires less production steps and enables more accurate alignment of the different parts composing the textile antenna. The proposed design covers all unlicensed national information infrastructure (U-NII) radio bands from $5.15 \mathrm{GHz}$ to 5.85 $\mathrm{GHz}$, making the antenna suitable for wireless communication through the 802.11ac standard. Finally, in Section 4 we introduce a holistic design framework for the co-design and co-optimization of the electronic circuits implementing communication, sensing and energy-harvesting functionality in autonomous textile nodes. The design procedure reconciles conflicting demands such as data rate, sensing capabilities, energy consumption, autonomy, cost, size and user comfort.

\section{Material characterization during assembly}

Consider a roll of substrate material being prepared in an assembly line for manufacturing textile antennas, as shown in Fig. 1 (right). To obtain precise information about each sample's permittivity and loss tangent in a non-destructive manner, a rigid test antenna with a sharp resonance peak (Fig. 1 (left)) is pressed against the material. The presence of the textile material on top of the antenna patch will produce a shift in the antenna's resonance frequency, for low-loss materials mainly depending on the MUT's permittivity. The width of the resonance peak will then serve to determine the loss tangent of the material. Before starting the characterization process, first electromagnetic field simulations are performed to construct a polynomial model relating the electromagnetic material properties to the frequencies at which the return loss crosses a certain threshold value, say $8 \mathrm{~dB}$, in the neighborhood of the resonance peak. Once this model is available, a rapid search will yield in real time the permittivity and loss tangent based on the corresponding measured return loss frequencies. More details are found in [4]. 


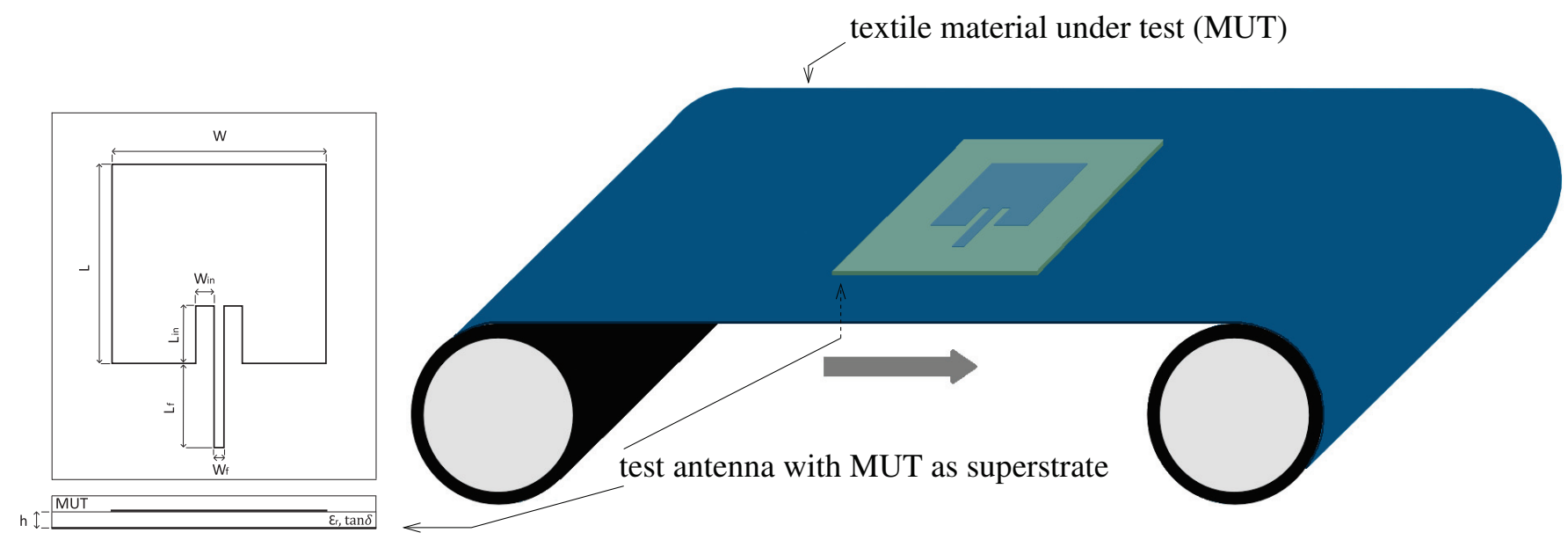

Figure 1. Just before assembly, an inset-fed patch antenna (left) is pressed against the material under test (MUT) (right). The shift in resonance frequency due to the MUT acting as superstrate yields the dielectric material properties.

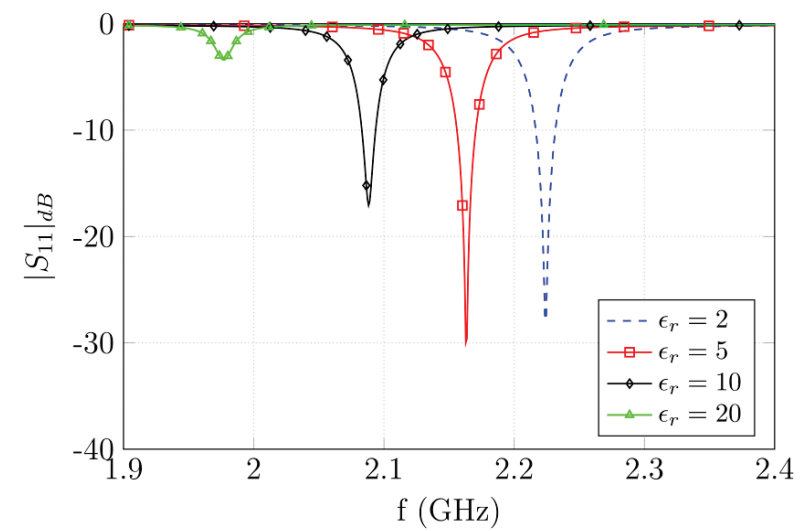

Figure 2. Shift in resonance frequency, due to a 2-mmthick superstrate with different permittivities and $\tan \delta=$ 0.005 .

As an example, we consider the effect of a 2-mm-thick material layer on the resonance peak of an inset-fed patch antenna designed to resonate at $2.25 \mathrm{GHz}$. This test antenna is implemented on a 0.508 -mm-thick I-Tera MT40 substrate $\left(\varepsilon_{r}=3.56, \tan \delta=0.0035\right)$. Fig. 2 shows the shift in resonance frequency, for different permittivity values, whereas Fig. 3 shows how the resonance peak broadens for increasing values of loss tangent. As a proof of principle, we have characterized a 2.01-mm-thick layer of EthyleneVinyl-Acetate (EVA) foam material. After constructing the polynomial expansions through full-wave electromagnetic simulations, a process that took about $15 \mathrm{~min}$, a permittivity of $\varepsilon_{r}=1.10$ and a loss tangent of $\tan \delta=0.040$ were characterized in less than $1 \mathrm{~s}$, based on the measured resonance peak of the test antenna with the foam serving as superstrate.

\section{Improved manufacturing process}

The conventional fabrication of wearable wireless systems, in general, and of textile antennas, in particular, is prone

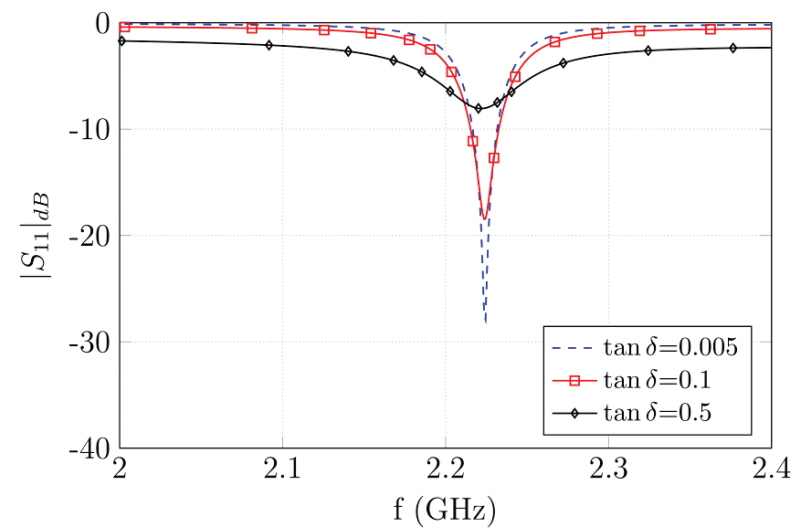

Figure 3. Broadening of the resonance peak, due to a 2mm-thick superstrate with different $\tan \delta$ values and $\varepsilon_{r}=2$.

to large variations in dimensions due to imprecise cutting and awkward alignment procedures. Moreover, most prototypes presented in research papers were constructed manually, a method that is time-consuming and costly. Typically, producing a similar prototype at large volumes and with high precision is not straightforward in an conventional industrial setting.

Substrate integrated waveguide (SIW) textile antennas provide excellent isolation between the antenna and the human body. Moreover, multimoding techniques may be applied to obtain ultra-wideband radiation characteristics. Yet, the accurate implementation of the via sidewalls may be difficult to achieve in a conventional mass production process. Moreover, the application of eyelets or embroidery may yield substrate compression and ripping. Therefore, we have developed a novel fabrication process for ultrawideband cavity-backed SIW textile antennas. The antenna cavity is cut for a computer-controlled laser out of a conventional textile foam, as shown in Fig. 4(a). Also a single sheet of electrotextile (Fig. 4(b)) is laser-patterned, such that it can be folded around the substrate. This sheet si- 


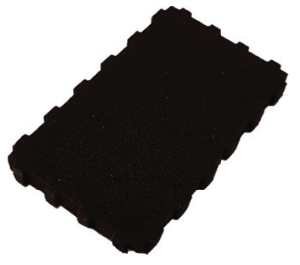

(a)

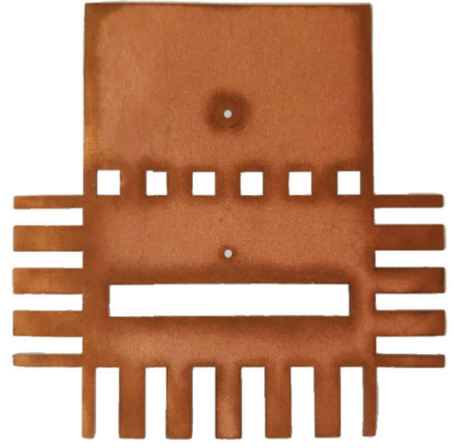

(b)

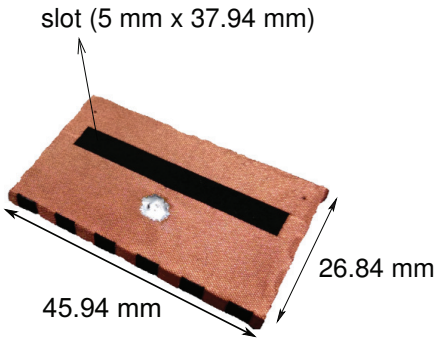

(c)

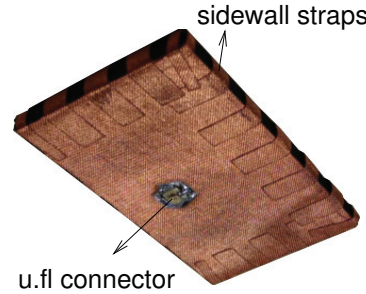

(d)

Figure 4. Manufacturing of an ultrawideband cavity-backed slot antenna based on a laser-cut (a) substrate and (b) electrotextile. Top view (c) showing antenna slot and Bottom view (d) showing u.fl connector serving as antenna feed.

multaneously implements the cavity slot through which the antenna radiates, the ground plane with a suitable footprint for the coaxial feed and the sidewalls formed by electrotextile slabs extending from the laser-cut patch. After assembly, during which a uf.l connector is attached to feed of the antenna, a compact, flexible and ultra-lightweight antenna module results, constructed in three simple steps, being first patterning the substrate, second the electrotextile and third the final assembly.

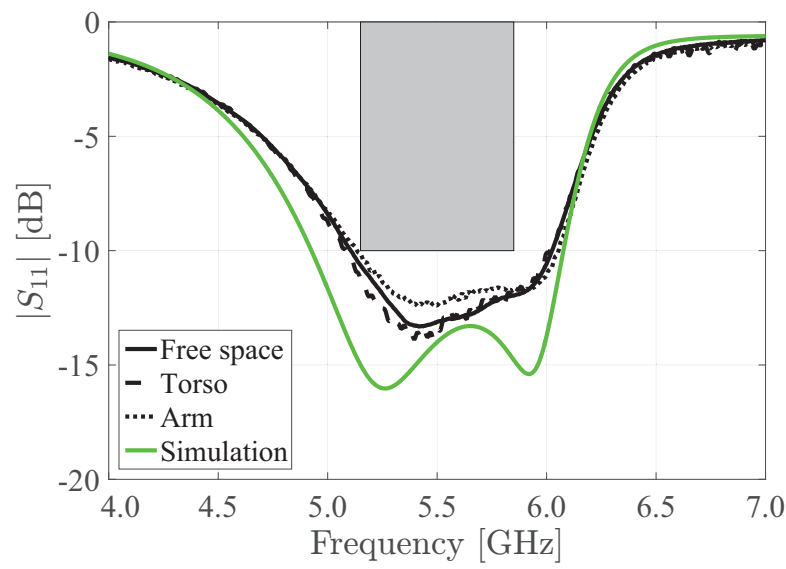

Figure 5. Simulated (free-space conditions) and measured return loss of a UNII-band cavity-backed SIW textile antenna, implemented according to the novel manufacturing procedure: performance in free space versus on body.

Fig. 4 shows a prototype implemented according to the new manufacturing technique. The antenna covers all unlicensed national information infrastructure (UNII) radio bands, extending from $5.15 \mathrm{GHz}$ to $5.85 \mathrm{GHz}$. Moreover, as shown in Fig. 5, this impedance bandwidth is maintained when the antenna is deployed on the human body. At $5.5 \mathrm{GHz}$, a maximum antenna gain of $6.7 \mathrm{dBi}$ is obtained in free-space conditions, compared to $4 \mathrm{dBi}$ when the antenna is deployed on the human body. For more details, we refer to reader to [5].

\section{Holistic textile wireless node design}

Smart wearable systems need to combine sensing, identification, localization and communication functionality with sufficient autonomy and user comfort. Satisfying these requirements in a reliable and cost-effective design remains highly challenging, in particular when direct integration into clothing is required. Therefore, we propose a novel procedure that co-optimizes all the different building blocks for synergetic optimal performance. This is achieved by first carefully selecting suitable materials and manufacturing techniques, as explained in previous sections. Next, the preliminary design of the critical building blocks starts, including the antenna system, the electronic circuits and the power supply system. In particular, a planar antenna, whose size is in the order of a wavelength to achieve sufficient radiation efficiency, is exploited as a platform for the active electronics and the energy harvesters. Moreover, by combining a highly power-efficient design with the application of energy harvesters, also sufficient autonomy is achieved and the use of bulky batteries is avoided. To characterize the effect of variability in the design parameters and uncertainty in the deployment conditions, also stochastic tools are integrated into the holistic design strategy.

To illustrate our strategy, we consider the design of an autonomous textile wireless sensor node operating in the 2.45GHz ISM band. Fig. 6, right, shows a block diagram of all necessary subsystems to implement sensing, processing, controlling and powering. This hardware is directly deployed on the planar antenna platform. Therefore, a circular microstrip patch antenna topology with shorting pins was adopted. Its radiation pattern is shaped such that reliable links are set up between access points placed in smart floors, ceiling and walls, and the node deployed on the human body. As schematically shown in Fig. 6, on top of the antenna patch, a flexible solar cell is positioned to harvest energy, which is then conditioned by the power management system placed on the antenna feed plane and subsequently stored in a flexible planar battery below this feed plane. More details about the design and dimensions of this textile wireless sensor node are found in [6]. 

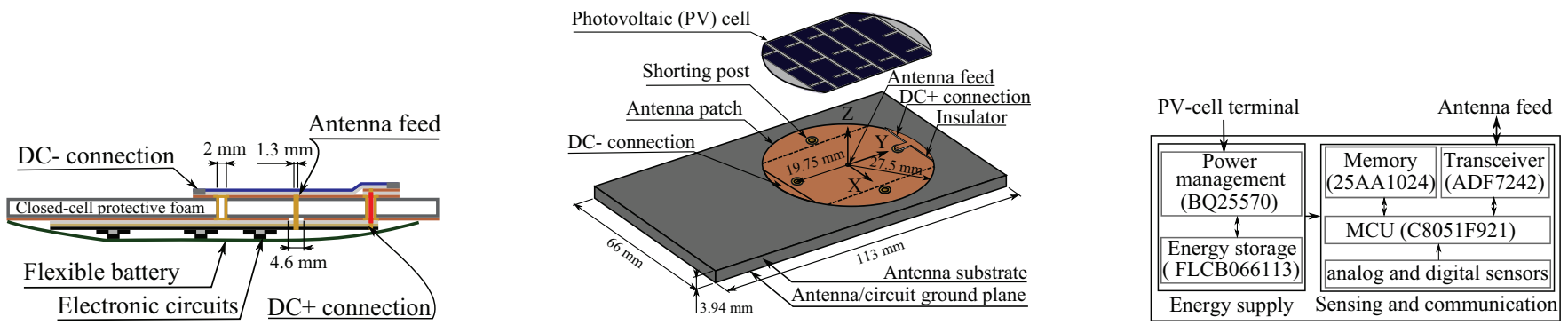

Figure 6. Autonomous wireless node operating in the $2.45 \mathrm{GHz}$ ISM band. Left: cross section showing the energy harvester on top of the antenna, the active electronics on the feedplane and the flexible battery deployed below the antenna system. Middle: Top view of the wireless node, with flexible solar cell shaped to fit on the antenna patch. Right: Block scheme showing different functional blocks.

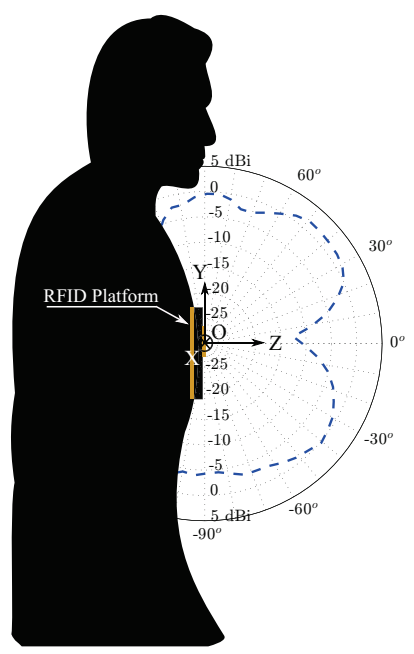

Figure 7. Radiation pattern of the wireless node deployed on the human body.

Measurements on a prototype have demonstrated that the antenna covers the complete $2.45 \mathrm{GHz}$ ISM-band in freespace conditions and when deployed on the human body. Moreover, the integration of photo-voltaic cell, electronic circuitry and flexible battery does not affect the antenna's radiation characteristics. When a sensor measurement is performed every minute, the node consumes $168 \mu \mathrm{W}$, offering at least 138 days of autonomy on battery power without harvesting any energy. Moreover, in an indoor environment the node may be interrogated wirelessly within a range of $23 \mathrm{~m}$. Fig. 7 shows the antenna gain of the wireless node when deployed on the human body. Note that the maximum radiation is oriented towards the walls, the floor and the ceiling, making this node ideal for wireless communication with smart surfaces. The maximum antenna gain amounts to $3.3 \mathrm{dBi}$ in free space before mounting the solar cell, $2.7 \mathrm{dBi}$ after integration of the solar cell and battery, and $2.5 \mathrm{dBi}$ when worn on the human body.

\section{Conclusion}

We have proposed some novel textile antenna design, material characterization and manufacturing procedures that can be directly integrated in conventional mass-production processes. These may be a first step for the successful market penetration of smart textiles.

\section{Acknowledgements}

This work was supported by the Research FoundationFlanders (FWO) (FWO) through the Excellence of Science (EoS) program

\section{References}

[1] P. Nepa, and H. Rogier, "Wearable Antennas for Off-Body Radio Links at VHF and UHF bands (below $1 \mathrm{GHz}$ ): Challenges, State-of-the-Art and Future Trends", IEEE Ant. and Propag. Mag., 57, 5, Oct. 2015, pp. 30-52, doi: 10.1109/MAP.2015.2472374.

[2] A. Kiourti and J. L. Volakis, "High-geometricalaccuracy embroidery process for textile antennas with fine details", IEEE Ant. and Wireless Propag. Letters, 14, 2015, pp. 1474-1477.

[3] S. Lemey, F. Declercq, and H. Rogier, "Textile antennas as hybrid energy harvesting platforms", Proc. of the IEEE, 102, 11, Nov. 2014, pp. 1833-1857.

[4] M. Rossi, S. Agneessens, H. Rogier, and D. Vande Ginste, "Assembly-line-compatible Electromagnetic Characterization of Wearable Antenna Substrates," IEEE Ant. and Wireless Propag. Letters, 16, 2017, pp. 1365-1368, doi: 10.1109/LAWP.2016.2636303.

[5] D. Van Baelen, S. Lemey, J. Verhaevert, H. Rogier, "A Novel Manufacturing Process for Compact, LowWeight and Flexible Ultra-Wideband Cavity Backed Textile Antennas", Materials, 11, 1, Jan. 2018, 17 pages, doi:10.3390/ma11010067.

[6] S. Lemey, S. Agneessens, P. Van Torre, K. Baes, J. Vanfleteren, and H. Rogier, "Wearable, flexible, light-weight modular RFID tag with integrated energy harvester", IEEE Trans. Microwave Theory Tech., 64, 7, Jul. 2016, pp. 2304-2314, doi: 10.1109/TMTT.2016.2573274. 